\title{
Efecto del entrenamiento en Agility en gran altitud en perros Border Collie en algunas variables electrocardiográficas: análisis preliminar
}

\author{
EfFect of training on AgIlity at high altitude in Border Collie dogs on SOME \\ ELECTROCARDIOGRAPHIC VARIABLES: PRELIMINARY ANALYSIS
}
Piero Vargas-Pinto ${ }^{1,4}$, Vladimir Galindo-Zamora ${ }^{1}$, Adriana Pedraza-Toscano ${ }^{2}$, Pedro Vargas-Pinto ${ }^{3}$

\section{Resumen}

\begin{abstract}
El objetivo del estudio fue conocer el efecto en reposo de algunas variables electrocardiográficas en perros Border Collie practicantes de la disciplina deportiva Agility en gran altitud (2600 msnm). Se evaluaron 15 perros (grupo atletas), pertenecientes a diferentes equipos de «agility» de la ciudad de Bogotá D.C., Colombia y 16 perros Border Collie no practicantes de actividades deportivas (grupo no atletas) de la misma ciudad. Las variables electrocardiográficas analizadas, en electrocardiografía de 5 min fueron: análisis de poder espectral con los componentes de alta y baja frecuencia y balance LF/ HF (simpático/vagal). Dentro del método de dominio de tiempo se determinó el promedio de RR (NN) y SDNN. En 100 latidos consecutivos se midió la duración de QRS y QT, corregido por la fórmula de Van de Water $(\mathrm{QTcV})$ y hallada su variabilidad por desviación estándar (SDQT). Se realizó observación de la dispersión de QT de cada uno de los individuos usando un gráfico de Poincaré. Los perros del grupo de atletas evidenciaron valores de HF más altos y de LF y LF/HF más bajos que el grupo de perros no atletas $(\mathrm{p}<0.05)$. Así mismo, los valores de RR y SDNN, longitud de QRS e intervalo QT fueron mayores en los perros atletas $(\mathrm{p}<0.05)$. No se encontraron diferencias entre los grupos en QTcV y SDQT. Los resultados obtenidos evidencian diferencias claras en varios componentes de la variabilidad de frecuencia cardíaca (VFC) entre perros Border Collie atletas y no atletas, con actividad diferenciada del sistema nervioso autónomo. El ejercicio y la altitud serían responsables de los hallazgos en QT y QRS.
\end{abstract}

Palabras clave: variabilidad de la frecuencia cardíaca; Agility; Border Collie; gran altura

\footnotetext{
${ }^{1}$ Departamento de Salud Animal, Facultad de Medicina Veterinaria y Zootecnia, Universidad Nacional de Colombia, Bogotá DC, Colombia

${ }^{2}$ Facultad de Medicina Veterinaria y Zootecnia, Universidad Antonio Nariño, Bogotá DC, Colombia

${ }^{3}$ Facultad de Ciencias Agropecuarias, Universidad de La Salle, Bogotá DC, Colombia

${ }^{4}$ E-mail: piavargaspi@unal.edu.co
}

Recibido: 7 de abril de 2017

Aceptado para publicación: 31 de agosto de 2017 
The aim of this study was to investigate the effect of the practice of the sport discipline of Agility at high altitude (2600 meters above sea level) on some electrocardiographic variables in Border Collie dogs at rest. Fifteen dogs (athlete group), belonging to different «Agility» teams from the city of Bogota D.C., Colombia and 16 Border Collie dogs, not trained in regular or athletic activities (non-athlete group) from the same city were evaluated. The electrocardiographic variables analyzed (5-min electrocardiography) were: spectral power density with high and low frequency components and LF/HF (sympathetic/vagal) balance. Within the time domain method, the mean RR (NN) and SDNN were determined. The duration of QRS and QT was corrected $(\mathrm{QTc})$ and its variability by standard deviation (SDQT) was measured in 100 consecutive beats. Observation of the QT dispersion of each individual was performed by Poincaré plot. The athlete group showed higher values of $\mathrm{HF}$ and of LF, and LF/HF lower than the non-athlete group $(\mathrm{p}<0.05)$. The values of RR and SDNN, QRS length and QT interval were also higher in athlete dogs $(\mathrm{p}<0.05)$. No differences were found between the groups in QTcV and SDQT. The results showed differences in several components of the heart rate variability (HRV) between Border Collie athletes and non-athletes, with differentiated activity of the autonomic nervous system. Exercise and altitude would be responsible for QT and QRS findings.

Key words: heart rate variability; Agility; Border Collie; high altitude

\section{INTRODUCCIÓN}

El atleta es generalmente un individuo sano y físicamente superior al resto de la población; sin embargo muchos atletas humanos que llevan a cabo actividades deportivas de resistencia presentan soplos cardíacos auscultables, bradiarritmias, vagotonía y evidencia electrocardiográfica de agrandamiento de cámaras (Zehender et al., 1990), lo que demuestra que un atleta expone su organismo a cambios fisiológicos y morfológicos que lo llevan al riesgo muy importante de sufrir arritmias y muerte súbita de origen cardíaco. En caninos también se han reportado anormalidades electrocardiográficas (Ponce Vázquez et al., 1998) y evidencias de remodelación eléctrica (Constable et al., 1994) en diversas actividades deportivas; no obstante, las consecuencias de una práctica deportiva con exigencia física submáxima como el Agility con exposición crónica a hipoxia hipobárica no han sido debidamente estudiadas.
Se han desarrollado múltiples herramientas para la evaluación de la función eléctrica cardíaca y una de ellas es la evaluación del tono autonómico a partir de la frecuencia cardíaca (FC) (Lahiri et al., 2008) que permite determinar los cambios que se producen antes, durante y después de concluido el ejercicio (Jouven et al., 2005), pero no permite la evaluación específica del control autonómico (Lewis y Short, 2010). Un complemento a esta herramienta es determinar su variabilidad en un tiempo determinado, capturar la oscilación de los intervalos entre latidos consecutivos, denominado esto como variabilidad de la frecuencia cardíaca (VFC) (Lahiri et al., 2008). La VFC está específicamente relacionada al volumen plasmático, actividad autonómica y posicionamiento corporal y su interpretación en entrenamiento está sujeta a algunos aspectos físicos del atleta; además de ser admitida como marcador sensible de alteraciones del sistema nervioso autónomo y riesgo de muerte de origen cardíaco (Hottenrott et al., 2006). 
En los últimos 30 años, la VFC determinada mediante el análisis de densidad del poder espectral ha ayudado al acercamiento no invasivo de la clínica al funcionamiento del sistema nervioso autónomo (SNA) (Piccirillo et al., 2009) y adaptación individual al entrenamiento (Plews et al., 2013; Dong, 1016). El segmento de alta frecuencia (HF 0.15-0.4 Hz) es la frecuencia de modulación del RR que está principalmente regulada por la inervación parasimpática (vagal) del corazón (Dong, 2016). Por su parte, el segmento de baja frecuencia (LF) del espectro se refiere a la regulación RR entre los 0.04 a $0.15 \mathrm{~Hz}$ y corresponde a la actividad de baroreceptores, que es a su vez dominada por la actividad simpática y parasimpática en conjunto (Oliveira et al., 2014a). Gracias a esta herramienta se ha demostrado que el entrenamiento de resistencia y las actividades de equipos en atletas humanos inducen una elevada modulación parasimpática (alta HF y bajo radio LF/HF) (Carter et al., 2003; Vanderlei et al., 2008). El análisis o método del dominio de tiempo (métodos estadísticos y métodos geométricos) es otra gran herramienta para la detección de la VFC. Dentro de los métodos estadísticos esta la SDNN (desviación estándar de todos los intervalos RR normales), que refleja todos los componentes cíclicos de la variabilidad en el periodo registrado y que puede ser medida en 24 horas $o$ en 5 minutos de registro (Electrophysiology, 1996).

En cuanto a la conducción eléctrica ventricular, la medición del intervalo QT implica la medición del tiempo de despolarización-repolarización de la célula miocárdica ventricular: el tiempo de duración del potencial de acción. La duración de QRS refleja el tiempo de despolarización de las mismas células. La duración de estos dos componentes del electrocardiograma puede ser modificada por alteración de la estructura ventricular en enfermedad o como consecuencia de entrenamiento físico repetido (Pelliccia et al., 1991, 2000). Todos estos marcadores electrocardiográficos mencionados son susceptibles de ser modificados gra- cias a la permanencia/entrenamiento en la gran altitud, debido a cambios estructurales y a cambios autonómicos cardíacos dentro de los procesos de aclimatación del individuo expuesto y variando en severidad según la altitud lograda, velocidad de ascenso y tiempo de permanencia (Perini et al., 1996; Glaus et al., 2003a; Monge Cassinelli y León Velarde, 2003; Sharshenova et al., 2006).

El propósito de este estudio fue determinar el efecto de la práctica deportiva Agility en gran altitud, en perros Border Collie pertenecientes a la Selección Colombia de Agility, respecto a los componentes HF, LF y LF/HF del análisis de densidad de poder espectral, RR (NN) y SDNN en análisis de dominio de tiempo y QRS, QT, QTcV y SDQT en electrocardiografía en reposo de cinco minutos y comparar los resultados con perros de la misma raza que no realizan actividades deportivas de alta competencia.

\section{Materiales y Métodos}

El estudio fue aprobado por el Comité de Bioética de la Facultad de Medicina Veterinaria y de Zootecnia de la Universidad Nacional de Colombia (Acta 10 del 1 de octubre de 2014). Se realizó un estudio transversal descriptivo en la ciudad de Bogotá DC, Colombia, la cual está ubicada a $2640 \mathrm{msnm}$, con presión atmosférica de $560 \mathrm{mmHg}$ y presión de $\mathrm{O}_{2}$ de $117.6 \mathrm{mmHg}$.

\section{Animales}

Se trabajó con 15 perros de la raza Border Collie de línea inglesa, machos y hembras, edad promedio $3.8 \pm 1.0$ años, practicantes de Agility, participantes del circuito local de competencias, homologados por la Asociación Club Canino Colombiano en los grados 2 y 3 (Certificación oficial de competencia/campeón nacional). Estos perros estuvieron en entrenamiento durante los dos años previos al examen electrocardiográfico. Se trabajó, además, con 16 perros no atletas 
de la raza Border Collie de línea inglesa, machos y hembras y de $3.18 \pm 1.2$ años promedio. Estos perros no hicieron parte de ninguna práctica deportiva profesional o habitual. Se excluyeron perros con historia reciente o evidencia de enfermedad sistémica y los perros de otras líneas de Border Collie.

El entrenamiento se realizaba de 2 a 3 veces por semana y mínimo 11 meses al año, con tiempos promedio de entrenamiento por sesión de 100 minutos. Esto incluía calentamiento de 10 a 20 minutos, velocidades altas combinadas con ejercicios de salto y estación intercalados con trote libre (protocolo de intervalos). El circuito local de competencias incluye aproximadamente 45 competencias por año.

\section{Electrocardiografía}

El examen electrocardiográfico fue realizado mediante Biopac MP $35^{\circledR}$ en canal de captura 1 para electrocardiografía con rango de 0.05 a $35 \mathrm{~Hz}$, a través de electrodos de «caimán» no traumáticos ubicados en miembro anterior izquierdo, piel de la región del olecranon, y en miembros posteriores en piel de la región del tendón patelar, humedecidos con gel para electrocardiografía. Cada individuo fue ubicado en decúbito lateral derecho, mediante restricción física suave, con miembros extendidos perpendiculares a la columna vertebral en cuarto tranquilo, aislado de ruidos, en horas con luz sol y siempre en presencia del propietario. Se capturó, mediante el programa BSL PRO 3.7 ${ }^{\circledR}$ (Biopac Systems, Inc), la derivación II durante mínimo 6 y máximo 10 min revisando la estabilidad del trazado durante el tiempo de registro. Los trazados fueron analizados mediante el programa AcqKnowledge 3.9 ${ }^{\circledR}$ (Biopac Systems, Inc), señalando 5 min de trazado estable y después de 30 segundos de comenzada la captura del electrocardiograma.

\section{Análisis de Poder Espectral}

Para el análisis del poder espectral de 5 min se establecieron las bandas de frecuen- cia de VLF en $0 \mathrm{~Hz}$ a $0.04 \mathrm{~Hz}$, LF $0.04 \mathrm{~Hz}$ a $0.15 \mathrm{~Hz}$, HF $0.15 \mathrm{~Hz}$ a $0.4 \mathrm{~Hz}$ y VHF $0.4 \mathrm{~Hz}$ a $3.0 \mathrm{~Hz}$ y el detector de QRS con umbral en $0.5 \mathrm{mv}$. Para el reporte de los valores LF y HF fueron utilizados los valores normalizados calculados por AcqKnowledge 3.9 ${ }^{\circledR}$ (Biopac Systems, Inc) mediante $\mathrm{LF}_{\mathrm{nu}}: \mathrm{LF} /$ (TP-VLF)*100 y HF ${ }_{\text {nu }}:$ HF/(TP-VLF)*100 (Chan et al., 2015). Se realizó inspección visual de los espectros de cada individuo.

\section{RR, NN y SDNN}

Los valores de los RR y NN posteriores se obtuvieron mediante análisis en $5 \mathrm{~min}$ consecutivos, al menos $30 \mathrm{~s}$ después de iniciado el registro, en ritmo sinusal previa visualización del trazado para detectar y descartar complejos ventriculares prematuros y otras anormalidades del ritmo. La medición de los RR se realizó de forma automática dentro del programa AcqKnowledge 3.9 ${ }^{\circledR}$ (Biopac Systems, Inc) y los datos obtenidos fueron transferidos a una hoja de cálculo de Excel para su análisis posterior. Los cálculos de las desviaciones estándar de los NN fueron realizados en hoja de cálculo de Excel ${ }^{\circledR}$.

\section{Intervalos QT (QTe) y Complejos QRS}

Para la medición de los complejos QRS (Figura 1a) y duración de intervalos QT (Figura 1b) fueron tomados 100 ciclos cardíacos consecutivos, al menos 30 segundos después de iniciado el registro, en ritmo sinusal y con visualización y diferenciación adecuada de cada onda. Se realizó en 100 ciclos para aumentar la precisión en los resultados, por encima de algunas sugerencias en la literatura (Hamlin et al., 2004).

La medición de los QT y QRS se realizó de forma manual dentro del programa AcqKnowledge 3.9 ${ }^{\circledR}$ (Biopac Systems, Inc) y los datos obtenidos transferidos a una hoja de cálculo de Excel ${ }^{\circledR}$ para su análisis posterior. Las desviaciones estándar de las mismas series de 100 ciclos fueron obtenidas para obtener la SDQT. Para la corrección de QT se utilizó la fórmula de Van de Water: 


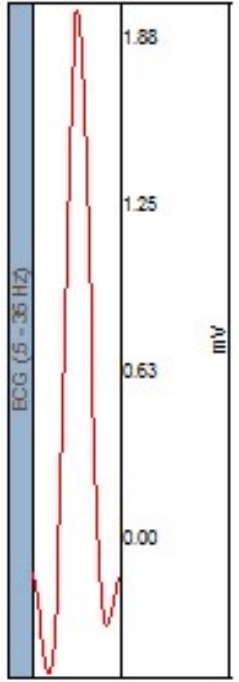

1a

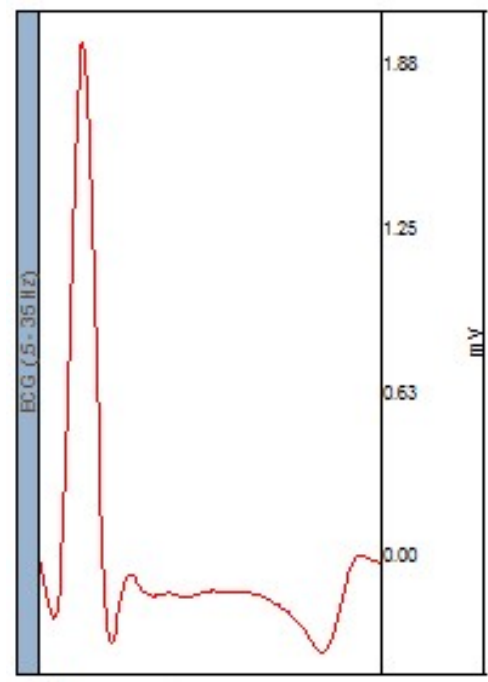

1b

Figura 1. Medición manual de QRS (1a) y QT (1b) mediante el programa AcqKnowledge 3.9. QRS se midió desde el inicio de Q hasta el final de S. QT se midió desde el inicio de $\mathrm{Q}$ hasta el final de $\mathrm{T}$. $\mathrm{ECG}=$ electrocardiograma; $\mathrm{mV}=$ milivoltio

QTc=QT-0.087(RR-1000) (Van de Water et al., 1989). Se realizó observación de la dispersión de QT por diagrama de Poincaré de la forma ya descrita (Hinterseer et al., 2010) para cada individuo de investigación, haciendo revisión visual de los patrones en cada uno (Kamen y Tonkin, 1995).

\section{Análisis Estadístico}

Para los test de normalidad se usó la prueba de Shapiro-Wilk. Para determinar la diferencia de medias entre valores con distribución normal se usó la prueba de $t$ no pareada. Para las que no presentaron distribución normal se enfrentaron mediante la prueba de Mann-Whitney. Los valores son presentados en media y desviación estándar, excepto en los casos señalados en los que se reporta mediana y rango. El valor de $p<0.05$ fue considerado significativo para todo el estudio. Se utilizó el paquete estadístico GraphPad Prism $4^{\circledR}$.

\section{Resultados}

Los perros del grupo de atletas evidenciaron valores de HF más altos (38.87 \pm 9.87 vs. $28.0 \pm 6.89 ; \mathrm{p}=0.0013)$ y de LF más bajos $(61.15 \pm 9.87$ vs. $72.0 \pm 6.89 ; \mathrm{p}=0.0013)(\mathrm{Fi}-$ gura 2). El balance LF/HF (1.70 \pm 0.61 vs. $2.75 \pm 0.92 ; p=0.0009)$ se encontró más bajo entre los perros atletas que en perros no atletas (Figura 3a). Los valores de RR (803.5 \pm 149.0 vs. $621.2 \pm 116.8$; $\mathrm{p}=0.0007)$ y $\mathrm{SDNN}$ (172.2, rango $89.90-414.4$ vs. 85.9 , rango 48.60-349.9; $\mathrm{p}=0.038$ ) fueron más altos en el grupo de atletas (Figura 3b), reflejando una mayor variación en los intervalos $\mathrm{RR}$.

La longitud de QRS $(64.21 \pm 3.81 \mathrm{vs}$. $57.22 \pm 8.04 ; \mathrm{p}=0.0048)$ e intervalo QT (229.6 \pm 22.68 vs. $209.5 \pm 17.61 ; \mathrm{p}=0.0098)$ fueron mayores en los perros atletas (Figura 4). No se encontraron diferencias entre los grupos en las variables QTc y SDQTc. El detalle de los resultados del análisis de densidad de po- 

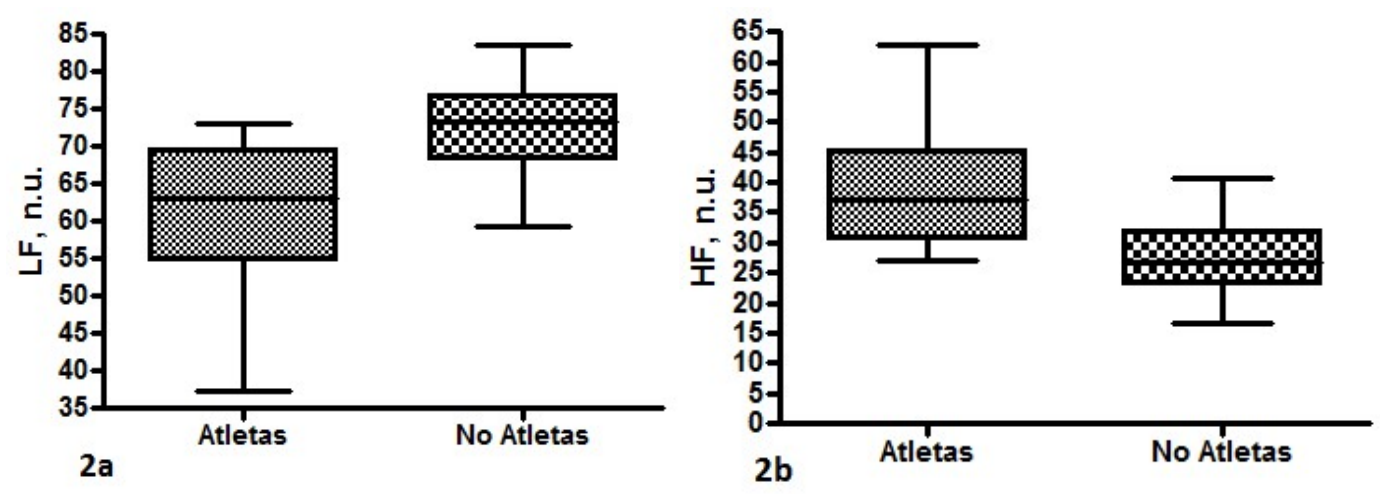

Figura 2. Comparativo del análisis de poder espectral para baja frecuencia LF (2a) y alta frecuencia HF (2b) entre perros atletas vs. no atletas a $2600 \mathrm{msnm}$. Los valores de LF fueron significativamente más bajos y los de HF más altos en perros atletas. n.u.= unidades normalizadas

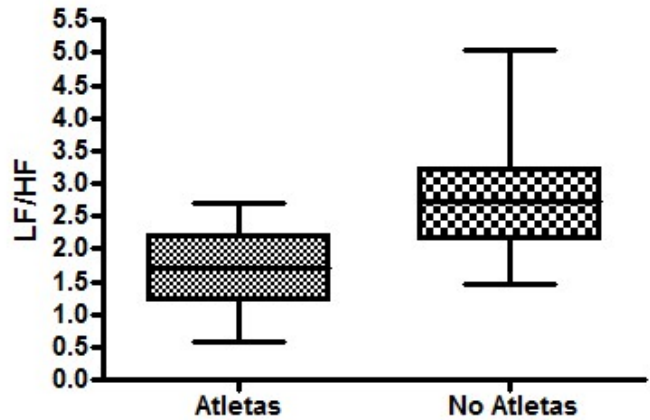

3a

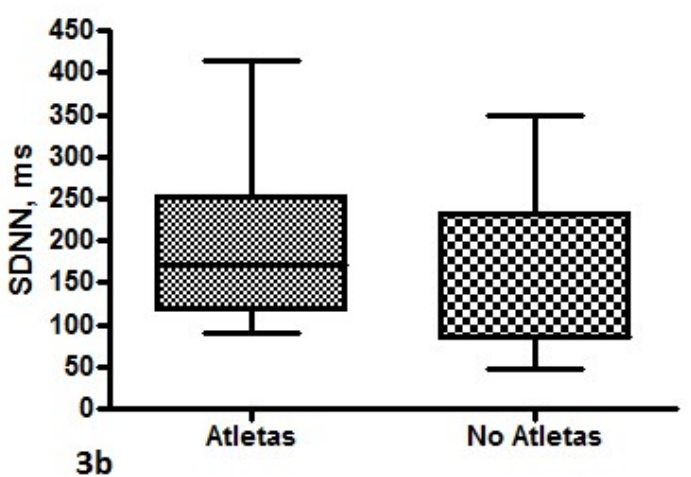

$3 b$

Figura 3. Comparativo del comportamiento del balance simpático/vagal (3a) y de la desviación estándar de los NN (SDNN) (3b). LF/HF= baja frecuencia/alta frecuencia; $\mathrm{ms}=$ milisegundos

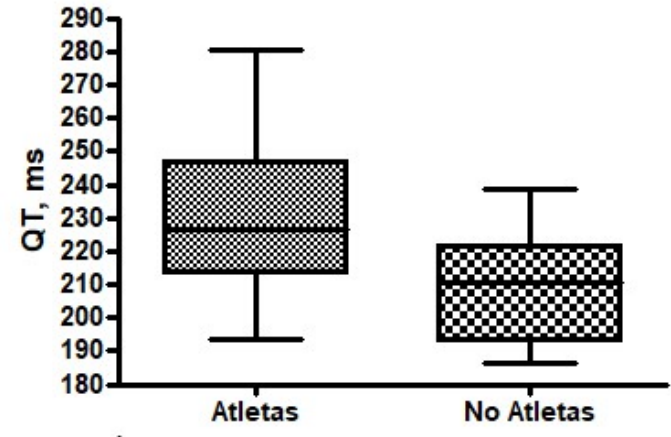

$4 a$

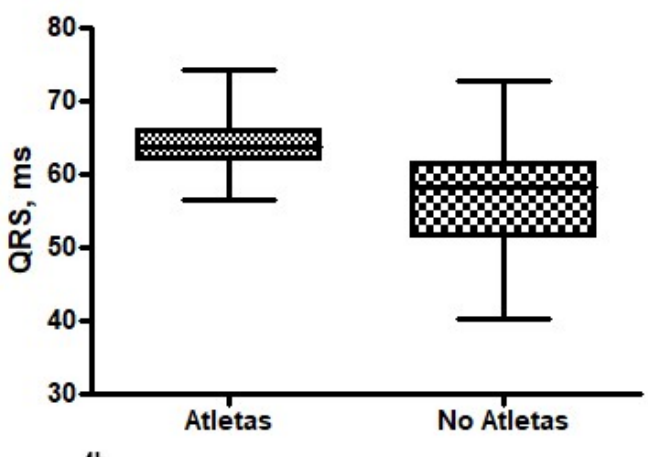

$4 \mathrm{~b}$

Figura 4. Comparativo del comportamiento estadístico de QT (a) y QRS (b) entre perros atletas vs. no atletas a $2600 \mathrm{msnm}$. Los perros atletas practicantes de Agility presentaron valores estadísticamente más altos en las dos mediciones. $\mathrm{ms}=$ milisegundos 

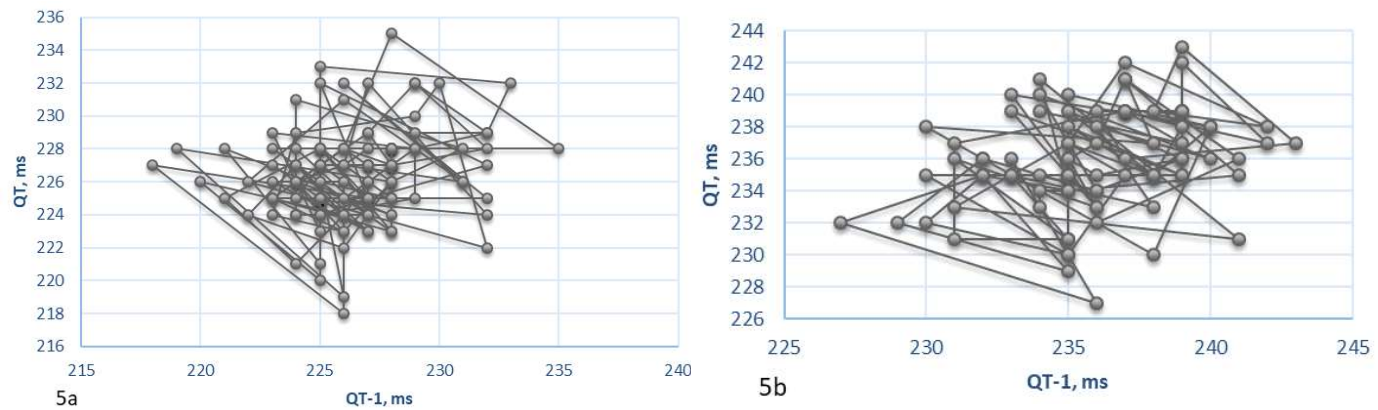

Figura 5. Comparativo de dispersión por gráfico Poincaré entre QT de un Border Collie atleta (a) y uno no atleta (b). $\mathrm{ms}=$ milisegundos

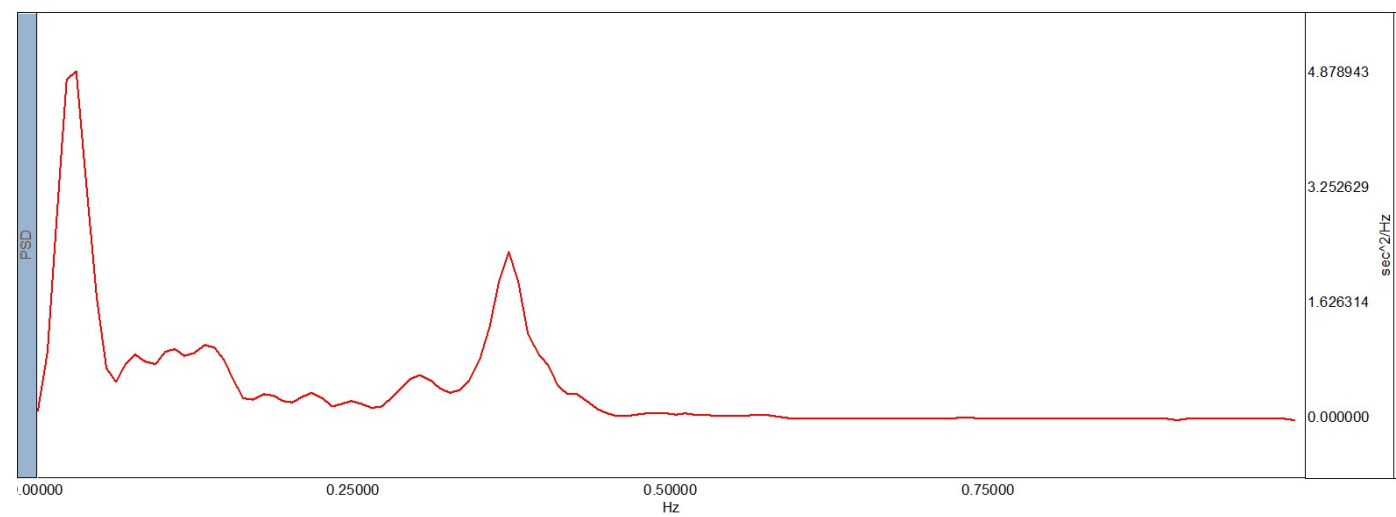

Figura 6. Densidad de poder espectral (PSD) de un perro Border Collie atleta. Predominio importante de VLF y LF con presencia de pico de HF (cerca de los 0.3-0.4 Hz). Hz= hertz

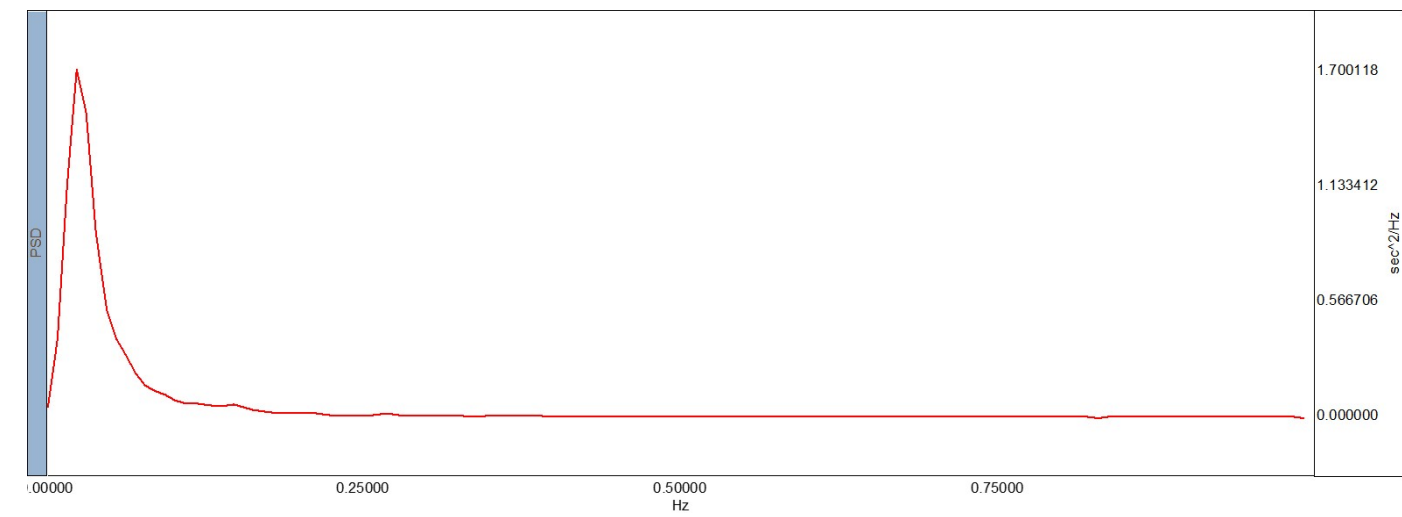

Figura 7. Densidad de poder espectral (PSD) de un perro Border Collie no atleta. Predominio de VLF y LF (0.04-0.15 Hz) en el espectro de este canino. $\mathrm{Hz}=$ hertz 
Cuadro 1. Resumen del análisis del poder espectral, NN y SDNN en perros Border Collie atletas $(\mathrm{n}=15)$ y perros no atletas $(\mathrm{n}=16)$ a $2600 \mathrm{msnm}$ (Bogotá, Colombia)

\begin{tabular}{lccccc}
\hline \multirow{2}{*}{ Medición } & \multirow{2}{*}{$95 \% \mathrm{CI}$} & \multirow{2}{*}{ Media/DE } & \multicolumn{2}{c}{ Percentil } & \multirow{2}{*}{$\mathrm{P}$} \\
\cline { 4 - 5 } & & & 25 & 75 & \\
\hline LF Atletas (u.n.) & $55.68-66.61$ & $61.15 \pm 9.871$ & 54.80 & 69.54 & 0.0013 \\
LF No Atletas & $68.33-75.67$ & $72.00 \pm 6.89$ & 68.21 & 76.83 & \\
LF/HF Atletas & $1.36-2.04$ & $1.70 \pm 0.61$ & 1.21 & 2.20 & 0.0009 \\
LF/HF No Atletas & $2.25-3.24$ & $2.75 \pm 0.92$ & 2.14 & 3.22 & \\
NN Atletas (ms) & $721.1-886.0$ & $803.5 \pm 149.0$ & 657.7 & 974.6 & \multirow{2}{*}{0.0007} \\
NN No Atletas & $558.9-683.4$ & $621.2 \pm 116.8$ & 515.2 & 704.1 & \\
SDNN Atletas\# (ms) & $137.5-234.9$ & $172.2(89.9-414.4)$ & 116.9 & 252.5 & 0.0098 \\
SDNN No Atletas\# & $87.86-189.3$ & $85.90(48.6-349.9)$ & 82.80 & 232.5 & \\
\hline
\end{tabular}

Valores indican media y desviación estándar, excepto para (\#) que indica mediana y rango. u.n.= unidades normalizadas, $\mathrm{ms}=$ milisegundos

Cuadro 2. Resumen del análisis de QT, QTcV, SDQT y QRS en perros Border Collie atletas $(\mathrm{n}=15)$ y perros no atletas $(\mathrm{n}=16)$ a 2600 msnm (Bogotá, Colombia)

\begin{tabular}{lccccc}
\hline \multirow{2}{*}{ Medición } & \multirow{2}{*}{$95 \% \mathrm{CI}$} & \multirow{2}{*}{ Media/DE } & \multicolumn{2}{c}{ Percentil } & \multirow{2}{*}{ P } \\
\cline { 1 - 3 } & & & 25 & 75 & \\
\hline QT Atletas (ms) & $217.0-242.2$ & $229.6 \pm 22.68$ & 213.3 & 247.0 & 0.0098 \\
QT No Atletas & $200.1-218.9$ & $209.5 \pm 17.61$ & 193.2 & 221.5 & \\
QTcV Atletas (ms) & $239.7-254.2$ & $246.9 \pm 13.07$ & 235.0 & 254.4 & 0.2852 \\
QTcV No Atletas & $236.4-248-0$ & $242.2 \pm 10.90$ & 232.4 & 248.9 & \\
SDQT Atletas\# (ms) & $3.49-5.25$ & $3.95(2.53-6.66)$ & 2.87 & 5.89 & \multirow{2}{*}{0.185} \\
SDQT No Atletas & $4.14-5.89$ & $5.02 \pm 1.64$ & 4.00 & 6.48 & \\
QRS Atletas (ms) & $62.10-62.33$ & $64.21 \pm 3.81$ & 62.00 & 66.00 & 0.0048 \\
QRS No Atletas & $52.93-61.51$ & $57.22 \pm 8.04$ & 51.60 & 61.50 & \\
\hline
\end{tabular}

der espectral (LF, HF, LF/HF) y de NN y SDNN se presentan en el Cuadro 1 y los de QT, QTcV, SDQT y QRS en el Cuadro 2. Los valores de QT en atletas fueron significativamente más altos, la dispersión visual fue similar y por SDNN no mostró diferencias significativas; además, se observó predominio de patrón de racimo abierto en los gráficos Poincaré para QT (Figura 5).

Las figuras 6 y 7 muestran el balance LF/HF de los perros atletas y los no atletas y los perfiles en el análisis de poder espectral. 


\section{Discusión}

En reporte previo (Vargas-Pinto et al., 2017) se describió el comportamiento de HF y LF $\left(\mathrm{ms}^{2}\right)$ en perros Border Collie atletas vs no atletas con banda de trote, donde los componentes de alta frecuencia fueron significativamente superiores en los perros atletas en el estado inmediatamente previo y en el descanso posterior al ejercicio con diferencias muy importantes en el comportamiento de los componentes de baja frecuencia con respecto a este nuevo estudio. El presente estudio (perros en reposo y decúbito lateral) mostró que el grupo de perros atletas practicantes de Agility evidenciaron valores significativamente más altos de los componentes HF y más bajos de los componentes LF que los perros no atletas.

En este estudio se encontró una mayor respuesta de la regulación parasimpática en perros atletas que en no atletas, hallazgo frecuente en atletas humanos de resistencia (Dixon et al., 1992; Billman et al., 2015), aunque no siempre encontrado (Scott et al., 2004). Esta respuesta es, además, dependiente del umbral respiratorio en humanos en algunas actividades deportivas (Cottin et al., 2004), algo que no está descrito para el Agility. Un número importante de estos cambios en la actividad autonómica cardíaca en los atletas se ha reportado como resultado de un aumento en la regulación por parte del sistema nervioso parasimpático, aunque también se han asociado estos cambios con la remodelación en el funcionamiento molecular del nodo sinoatrial (Boyett et al., 2013), 1levando a una disminución en la densidad de las $I_{f}$ (D'Souza et al., 2014), permitiendo otra interpretación probable a estos resultados en humanos y animales entrenados en actividad física. Esta última teoría fue refutada por el hallazgo de una mayor expresión de canales $\mathrm{HCN} 4$, proteína responsable del tránsito en las corrientes $I_{f}$, que no explicaría la bradicardia que presentan los individuos entrenados (Billman et al., 2015). Por otro lado, algunos autores sugieren que tanto incremen- tos como decrementos en la variabilidad podrían ser asociadas con mala adaptación al entrenamiento (Plews et al., 2013).

Al observar la predominancia de los valores LF en los perros no atletas, el balance LF/HF de los perros atletas y los no atletas y los perfiles en el análisis de poder espectral (Figuras 6 y 7), es posible deducir que, en los dos grupos con mayor importancia en los no atletas, existe un menor predominio del control parasimpático en la VFC. Es de especial interés anotar que los Border Collie de la línea inglesa de trabajo presentan grandes rasgos de ansiedad cuando se les restringe su movimiento, lo que podría condicionar una mayor actividad simpática durante la toma de los trazados electrocardiográficos.

Los resultados obtenidos en este estudio, aunque con diferencias estadísticas significativas entre grupos, muestra gran diferencia con los reportados en perros sanos de otras razas en los componentes HF, LF y LF/ HF (Oliveira et al., 2014a). En el presente estudio, los perros Border Collie atletas muestran una tendencia hacia una menor regulación parasimpática y un mayor radio LF/HF (simpático vagal) que se asemejan a los perros en estadio C y D (Atkins et al., 2009) de degeneración valvular mixomatosa mitral (DVMM), según los resultados reportados. También se encuentran amplias diferencias entre el radio LF/HF de este estudio con el reporte de Bogucki y Noszczyk-Nowak (2015) en 50 perros de varias razas mostrando en el presente estudio valores notoriamente más altos.

Los resultados de la SDNN fueron significativamente más bajos en perros no atletas que en el grupo de atletas, reflejando también un menor control parasimpático en la VFC de los perros que no practican actividad física regular bajo las condiciones de este estudio. Estos resultados fueron también muy diferentes a los obtenidos en el estudio realizado por Bogucki y Noszcyk-Nowak (2015), posiblemente debido a que en dicho estudio se trabajó con perros sanos de varias razas. 
No se dispone de estudios previos de VFC en gran altitud en perros con fines de comparación; sin embargo, estudios en humanos han mostrado que la altitud modifica la VFC de formas diferentes, según edad y género (Sharshenova et al., 2006), y que la exposición a grandes altitudes decrece significativamente la actividad nerviosa parasimpática (Long et al., 2006), llevando hacia una dominancia del simpático (Perini et al., 1996). Al estar los perros de ambos grupos expuestos a hipoxia hipobárica crónica y resultante hipoxemia (Glaus et al., 2003; Forero-López et al., 2006), se deduce que estos procesos ocurran de forma similar en la especie canina y podrían tener parte de la responsabilidad en las diferencias en los valores de VFC.

Otro hallazgo importante fue que la longitud promedio de QRS fue significativamente mayor en los perros entrenados en Agility (Figura 4b), lo cual sugiere un fenómeno de remodelación cardíaca como consecuencia de la actividad deportiva que realizan estos individuos. Estos hallazgos que diferencian a los perros atletas de los no atletas de una misma raza fueron reportados en perros de trineo de Alaska (Constable et al., 1994), y se evidenciaron en siete meses de entrenamiento asociados al incremento del peso cardíaco (Constable et al., 2000).

Los valores promedio de QRS en los dos grupos fueron menores a los perros de trineo de Alaska (antes y después de entrenamiento) (Constable et al., 2000), pero ligeramente mayores que en estos perros cuando no estaban entrenados o con poco entrenamiento (Constable et al., 1994). También fueron mayores a los QRS en perros Beagles (Hanton y Rabemampianina, 2006) y en perros Labrador, Pastor Alemán y Golden Retriever sanos (Mukherjee et al., 2015), incluso en el grupo de caninos no atletas. Estos resultados estarían relacionados con las diferencias ecocardiográficas encontradas entre la raza Border Collie y otras razas, específicamente en la dimensión interna ventricular izquierda (LVID) (Jacobson et al., 2013). Sumado a lo anterior, se podría inferir que se estaría presentando una leve remodelación ventricular derecha inducida por la hipoxia hipobárica de los $2600 \mathrm{msnm}$ en la que viven estos perros, ya que se ha reportado hipertensión arterial pulmonar leve en caninos que viven a $2300 \mathrm{msnm}$ (Glauss et al., 2003a,b).

De forma similar a los resultados de QRS, los hallazgos de QT fueron significativamente más altos en los perros del grupo de atletas que en los no atletas (Figura 4a). En la interpretación de este resultado se requiere tener en cuenta lo enunciado en cuanto la remodelación cardíaca como consecuencia del entrenamiento físico crónico, así como la correlación negativa reportada entre QT y frecuencia cardíaca en estudios previos (Oliveira et al., 2014b), que se puede inferir más alta en el grupo de no atletas por las diferencias en los RR entre los dos grupos. Los valores de QT fueron corregidos mediante la fórmula de corrección de Van de Water (Van de Water, 1989) y a diferencia de los valores crudos, no mostraron diferencias estadísticas entre grupos. Esta fórmula ha proveído resultados óptimos y consistentes en la corrección de QT en estudios farmacológicos (King et al., 2006; Soloviev et al., 2006) y clínicos (Oliveira et al., 2014a). Esta diferencia de resultados en el mismo estudio se debe a la mayor corrección que realiza la fórmula de Van de Water en las frecuencias cardíacas más altas de los perros no atletas.

Los resultados obtenidos en los valores de QT y QTcV en los dos grupos de interés son superiores a los reportados por Oliveira et al. (2014b) en perros de las razas Pastor Alemán, Bóxer, Poodle y Pit Bull Terrier, así como a los perros Beagle (Hanton y Rabemampianina, 2006), pero muy similares, al estudio de Mukherjee et al. (2015) realizado en perros no atletas de razas Labrador, Pastor Alemán y Golden Retriever. 
Los valores de SDQT como medida de la variación de las medias de QT no mostraron diferencias significativas entre atletas y no atletas, y refleja, para esta modalidad de medición, la similitud del proceso despolarización-repolarización con mayor importancia para el último entre los dos grupos de investigación. No se encontraron estudios previos donde se considere SDQT en caninos o caninos atletas. Por otro lado, los gráficos de Poincaré se portaron previsiblemente de forma similar a los resultados de SDQT (Figura 5) con un patrón de racimo abierto típico de los datos con distribución normal (Kamen y Tonkin, 1995) y con clasificación visual basada en la clasificación de $R R$ usado en el estudio referenciado. Se hace necesario someter los intervalos QT a otros modelos de determinación de la variabilidad.

\section{Conclusiones}

- La práctica del Agility genera diferencias en la regulación autonómica y remodelación eléctrica en los perros. Se presenta aumento en los componentes HF y disminución de los componentes LF y del balance simpático/vagal.

- Existen diferencias marcadas en la densidad espectral (LF, HF, LF/HF) y SDNN con otros estudios realizados con otras razas de perros y en diferentes condiciones de presión barométrica.

- Hallazgos similares son descritos para los valores de QT, QTcV y QRS.

\section{Agradecimientos}

Los autores agradecen la colaboración prestada por parte de los integrantes de la Selección Colombia de Agility, específicamente a los clubes de la ciudad de Bogotá D.C., Colombia.

\section{Literatura Citada}

1. Atkins C, Bonagura J, Ettinger S, Fox P, Gordon S, Haggstrom J, Hamlin $\boldsymbol{R}$, et al. 2009. Guidelines for the diagnosis and treatment of canine chronic valvular heart disease. J Vet Intern Med 23: 1142-1150. doi: 10.1111/j.19391676.2009.-0392.x

2. Billman GE, Cagnoli KL, Csepe T, Li N, Wright P, Mohler PJ. Fedorov VV. 2015. Exercise training-induced bradycardia: evidence for enhanced parasympathetic regulation without changes in intrinsic sinoatrial node function. J Appl Physiol 118: 1344-1355. doi: 10.1152/japplphysiol.01111.2014

3. Bogucki S, Noszczyk-Nowak A. 2015. Short-term heart rate variability (HRV) in healthy dogs. Pol J Vet Sci 18: 307312. doi: $10.1515 /$ pjvs-2015-0040

4. Boyett MR, D'Souza A, Zhang $H$, Morris GM, Dobrzynski H, Monfredi O. 2013. Viewpoint: is the resting bradycardia in athletes the result of remodeling of the sinoatrial node rather than high vagal tone? J Appl Physiol 114: 1351-1355. doi: 10.1152/japplphysiol.01126.2012

5. Carter JB, Banister E, Blaber AP. 2003. Effect of endurance exercise on autonomic control of heart rate. Sports Med 33: 33-46. doi: 10.2165/00007256200333010-00003

6. Chan YH, Tsai WC, Shen C, Han S, Chen LS, Lin SF, Chen PS. 2015. Subcutaneous nerve activity is more accurate than the heart rate variability in estimating cardiac sympathetic tone in ambulatory dogs with myocardial infarction. Heart Rhythm 12: 1619-1627. doi: 10.1016/j.hrthm.2015.03.025

7. Constable PD, Hinchcliff KW, Olson J, Hamlin RL. 1994. Athletic heart syndrome in dogscompetinginalong-distance sled race. JAppl Physiol 76: 433-438. 
8. Constable PD, Hinchcliff $\mathrm{KW}$, Olson J, Stepien RL. 2000. Effects of endurance training on standard and signal-averaged electrocardiograms of sled dogs. Am J Vet Res 61: 582-588. doi: 10.2460/ajvr.2000.61.582

9. Cottin F, Médigue C, Leprêtre PM, Papelier Y, Koralsztein JP, Billat V. 2004. Heart rate variability during exercise performed below and above ventilatory threshold. Med Sci Sports Exerc 36: 594-600.

10. Dixon EM, Kamath MV, McCartney $N$, Fallen EL. 1992. Neural regulation of heart rate variability in endurance athletes and sedentary controls. Cardiovasc Res 26: 713-719. doi: $10.1093 / \mathrm{cvr} / 26.7 .713$

11. Dong JG 2016. The role of heart rate variability in sports physiology. Exp Ther Med 11: 1531-1536. doi: 10.3892/ etm.2016.3104

12. D'Souza A, Bucchi A, Johnsen AB, Logantha SJRJ, Monfredi O, Yanni $J$, Prehar S, et al. 2014. Exercise training reduces resting heart rate via downregulation of the funny channel HCN4. Nat Commun 5: 3775. doi: 10.1038/ncomms4775

13. Electrophysiology. 1996. Task Force of the European Society of Cardiology and the North American Society of Pacing and Electrophysiology. Heart rate variability: standards of measurement, physiological interpretation and clinical use. Circulation 93: 1043-1065. doi: 10.1161/01.CIR.93.5.1043

14. Glaus TM, Hauser K, Hässig M, Lipp B, Reusch CE. 2003a. Non-invasive measurement of the cardiovascular effects of chronic hypoxaemia on dogs living at moderately high altitude. Vet Rec 152: 800-803.

15. Glaus TM, Hässig M, Baumgartner C, Reusch CE. 2003b. Pulmonary hypertension induced in dogs by hypoxia at different high-altitude levels. Vet Res Commun 27: 661-670. doi: 10.1023/ A: 1027380614534
16. Hamlin RL, Kijtawornrat A, Keene $\boldsymbol{B W}$. 2004. How many cardiac cycles must be measured to permit accurate $\mathrm{RR}, \mathrm{QT}$, and QTc estimates in conscious dogs? J Pharmacol Toxicol Methods 50: 103-108. doi: 10.1016/j.vasen.2004.03.013

17. Hanton G, Rabemampianina Y. 2006. The electrocardiogram of the Beagle dog: reference values and effect of sex, genetic strain, body position and heart rate. Lab Anim 40: 123-136. doi: 10.1258/ 002367706776319088

18. Hinterseer M, Beckmann BM, Thomsen MB, Pfeufer A, Ulbrich M, Sinner MF, Perz S, et al. 2010. Usefulness of short-term variability of QT intervals as a predictor for electrical remodeling and proarrhythmia in patients with nonischemic heart failure. Am J Cardiol 106: 216-220. doi: 10.1016/ j.amjcard.2010.02.033

19. Hottenrott K, Hoos O, Esperer HD. 2006. Heart rate variability and physical exercise. Current status. Herz 31: 544552. [in German]. doi: 10.1007/s00059006-2855-1

20. Jacobson JH, Boon JA, Bright JM. 2013. An echocardiographic study of healthy Border Collies with normal reference ranges for the breed. $J$ Vet Cardiol 15: 123-130. doi: 10.1016/ j.jvc.2012.12.005

21. Jouven X, Empana JP, Schwartz PJ, Desnos M, Courbon D, Ducimetière P. 2005. Heart-rate profile duringe exercise as a predictor of sudden death. N Engl J Med 352: 1951-1958. doi: 10.1056/NEJMoa043012

22. Kamen PW, Tonkin AM. 1995. Application of the Poincare plot to heart rate variability: a new measure of functional status in heart failure. Aust NZ J Med 25: 18-26. doi: 10.1111/j.14455994.1995.tb00573.x

23. King A, Bailie M, Olivier NB. 2006. Magnitude of error introduced by application of heart rate correction formulas to the canine QT interval. Ann 
Noninvasive Electrocardiol 11:289-298. doi: 10.1111/j.1542-474X.2006.00120.x

24. Lahiri MK, Kannankeril PJ, Goldberger JJ. 2008. Assessment of autonomic function in cardiovascular disease: physiological basis and prognostic implications. J Am Coll Cardiol 51: 1725-1733. doi: 10.1016/ j.jacc.2008.01.038

25. Lewis MJ, Short AL. 2010. Exercise and cardiac regulation: what can electrocardiographic time series tell us? Scand J Med Sci Sports 20: 794-804. doi: 10.1111/j.1600-0838.2010.01150.x.

26. Long M, Qin J, Huang L, Tian K, Yu $S, Y u$ Y. 2006. Comparison of heart rate variability in healthy young men during exposure to different altitudes. Sheng $\mathrm{Wu}$ Yi Xue Gong Cheng Xue Za Zhi 23: 1195-1197. [in Chinese].

27. Forero-López JH, Lozano-Martínez PA, Camargo-Roncancio BO. 2006. Parámetros fisiológicos en caninos pre y post competencia de Agility en Bogotá, Colombia. Rev Med Vet 12: 57-71. doi: 10.19052/mv.2053

28. Monge Cassinelli C, León Velarde F. 2003. El reto fisiológico de vivir en los Andes. Lima, Perú: IFEA. 435 p.

29. Mukherjee J, Das PK, Ghosh PR, Banerjee D, Sharma T, Basak D, Sanyal S. 2015. Electrocardiogram pattern of some exotic breeds of trained dogs: a variation study. Vet World 8: 1317-1320. doi: 10.14202/vetworld.2015.1317-1320

30. Oliveira MS, Muzzi RAL, Araújo RB, Muzzi LAL, Ferreira DF, Silva EF. 2014a. Heart rate variability and arrhythmias evaluated with Holter in dogs with degenerative mitral valve disease. Arq Bras Med Vet Zootec 66: 425-432. doi: 10.1590/1678-41626097

31. Oliveira MS, Muzzi RAL, Muzzi LAL, Cherem M, Mantovani MM. $2014 b$. QT interval in healthy dogs: which method of correcting the QT interval in dogs is appropriate for use in small animal clinics? Pesqu Vet Bras 34: 469-472. doi: 10.1590/S0100-736X2014000500014
32. Pelliccia A, Maron BJ, Spataro A, Proschan MA, Spirito P. 1991. The upper limit of physiologic cardiac hypertrophy in highly trained elite athletes. N Engl J Med 324: 295-301. doi: 10.1056/NEJM199101313240504

33. Pelliccia A, Maron BJ, Culasso F, Paolo FMD, Spataro A, Biffi A, Caselli G, Piovano P. 2000. Clinical significance of abnormal electrocardiographic patterns in trained athletes. Circulation 102: 278-284. doi: 10.1161/01.CIR.102.3.278

34. Perini R, Milesi S, Biancardi L, Veicsteinas A. 1996. Effects of high altitude acclimatization on heart rate variability in resting humans. Eur J Appl Physiol Occup Physiol 73: 521-528. doi: 10.1007/BF00357674

35. Piccirillo G, Ogawa M, Song J, Chong VJ, Joung B, Han S, Magri D, et al. 2009. Power spectral analysis of heart rate variability and autonomic nervous system activity measured directly in healthy dogs and dogs with tachycardiainduced heart failure. Heart Rhythm 6: 546-552. doi: 10.1016/j.hrthm.2009.01.006

36. Plews DJ, Laursen PB, Stanley J, Kilding AE, Buchheit M. 2013. Training adaptation and heart rate variability in elite endurance athletes: opening the door to effective monitoring. Sports Med 43: 773-781. doi: 10.1007/ s40279-013-0071-8

37. Ponce Vázquez J, Pascual Gómez F, Alvarez Badillo A, Dolz Luna JF, Rodríguez Rodríguez LP. 1998. Arritmias cardíacas inducidas por el ejercicio dinámico máximo de corta duración (sprint): estudio en el galgo Greyhound. Rev Esp Cardiol 51: 559-565.

38. Scott AS, Eberhard A, Ofir D, Benchetrit G, Dinh TP, Calabrese P, Lesiuk V, Perrault H. 2004. Enhanced cardiac vagal efferent activity does not explain training-induced bradycardia. Auton Neurosci 112: 60-68. doi: 10.1016/ j.autneu.2004.04.006 
39. Sharshenova AA, Majikova EJ, Kasimov OT, Kudaiberdieva G 2006. Effects of gender and altitude on shortterm heart rate variability in children. Anadolu Kardiyol Derg 6: 335-339.

40. Soloviev MV, Hamlin RL, Barrett RM, Chengelis CP, Schaefer GJ. 2006. Different species require different correction factors for the QT interval. Cardiovasc Toxicol 6: 145-157. doi: 10.1385/CT:6:2:145

41. Van de Water A, Verheyen J, Xhonneux R, Reneman RS. 1989. An improved method to correct the QT interval of the electrocardiogram for changes in heart rate. J Pharmacol Methods 22: 207-217. doi: 10.1016/01605402(89)90015-6
42. Vanderlei LCM, Silva RA, Pastre CM, Azevedo FM, Godoy MF. 2008. Comparison of the Polar S810i monitor and the ECG for the analysis of heart rate variability in the time and frequency domains. Braz J Med Biol Res 41: 854-859. doi: 10.1590/ S0100-879X200800-5000039

43. Vargas-Pinto P, Arango S, Rodríguez V, Rivas J, Vargas-Pinto P. 2017. Frecuencia cardiaca y su variabilidad en caninos de Agility en gran altura. Rev Inv Vet Perú 28: 236-241. doi: http:// dx.doi.org/10.15381/rivep.v28i2.13071

44. Zehender M, Meinertz, T, Keul J, Just H. 1990. ECG variants and cardiac arrhythmias in athletes: Clinical relevance and prognostic importance. Am Heart J 119: 1378-1391. 